\title{
ANALYTICITY AND CONTINUATION OF CERTAIN FUNCTIONS OF TWO COMPLEX VARIABLES ${ }^{1}$
}

\author{
CARL H. FITZGERALD
}

\begin{abstract}
This paper shows that the satisfaction of a certain quadratic relation is a sufficient condition that a continuous, symmetric function of two complex variables on a domain be analytic and be continuable to a particular larger domain. This quadratic relation is of the same type as that involved in the Grunsky inequalities.
\end{abstract}

In proving a generalization of the Grunsky inequalities, Bergman and Schiffer [3] announced a theorem on analytic continuation of a function of two complex variables. In extending the Grunsky inequalities in another way, Alenicyn [1] found this theorem on continuation useful. The purpose of this note is to strengthen the Bergman-Schiffer theorem to be more natural for both applications and to provide a proof that is more direct than the formal computation in the original proof.

Suppose $D$ and $\mathcal{G}$ are bounded domains, and $\mathcal{G}$ is contained in $D$. Let $\int_{\mathcal{D}} \cdot d A_{z}$ denote area integration as $z$ ranges over $\mathfrak{D}$. Let $K \mathcal{D}(z, \bar{\zeta})$ be the Bergman kernel function [2] for the domain $D$.

THEOREM. If $V(z, \zeta)$ is a symmetric, continuous, complex-valued

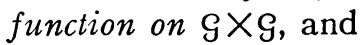

$$
\left|\int_{\mathcal{S}} \int_{S} V(z, \zeta) \overline{\phi(z)} \overline{\phi(\zeta)} d A_{z} d A_{\zeta}\right| \leqq \int_{\mathcal{S}} \int_{\mathcal{S}} K \mathfrak{D}(z, \bar{\zeta}) \overline{\phi(z)} \phi(\zeta) d A_{z} d A_{\zeta}
$$

for all continuous, complex-valued function $\phi$ with compact support in $\mathcal{G}$, then $V(z, \zeta)$ is analytic in $\mathcal{G} \times \mathcal{G}$ and can be continued onto $D \times D$.

Proof. Let $G$ be a subdomain of $\mathcal{G}$ such that the closure $\bar{G}$ is contained in $g$. There exists a complete orthonormal system of analytic functions $\left\{\phi_{n}\right\}_{n=1}^{\infty}$ on $D$, which is also orthogonal on $G,[2]$. Let

$$
k_{n}^{2}=\int_{G} \phi_{n}(z) \overline{\phi_{n}(z)} d A_{z} \quad \text { for } n=1,2, \cdots .
$$

Received by the editors October 7, 1969.

AMS Subject Classifications. Primary 3028, 3086, 3235; Secondary 3009, 3042.

Key Words and Phrases. Grunsky inequalities, Bergman kernel function, analytic continuation, two complex variables, doubly orthogonal functions.

1 This work was supported in part by the Air Force Grant AFOSR-68-1514. 
Then $\left\{\phi_{n}(z) / k_{n}\right\}_{n=1}^{\infty}$ is an orthonormal system on $G$, but is not necessarily a complete system.

Let $Q_{n m}$ be defined by

$$
k_{n} k_{m} \mathrm{Q}_{n m}=\int_{G} \int_{G} V(z, \zeta) \overline{\phi_{n}(z)} \overline{\phi_{m}(\zeta)} d A_{z} d A_{\zeta} .
$$

Since $V(z, \zeta)$ is continuous on $\bar{G} \times \bar{G}, \int_{G} \int_{G}|V(z, \zeta)|^{2} d A_{z} d A_{\zeta}<\infty$. Then by the usual argument, [2]

$$
\sum_{n, m=1}^{\infty} \alpha_{n m} \phi_{n}(z) \phi_{m}(\zeta)
$$

converges uniformly on compact subsets of $G$. It is now shown that the series converges to $V(z, \zeta)$.

Suppose $\Gamma_{1}(z)$ is a continuous function on $G$, has $\int_{G}\left|\Gamma_{1}(z)\right|^{2} d A_{z}$ $<\infty$, and is orthogonal to $\phi_{n}$ on $G$ for $n=1,2, \ldots$. Let $\Gamma_{2}(z)$ be any continuous function on $\bar{G}$, and $\lambda$ be a real number. By using the symmetry of $V(z, \zeta)$,

$$
\begin{aligned}
& \left|\int_{G} \int_{G} V(z, \zeta)\left[\overline{\Gamma_{1}(z)+\lambda \Gamma_{2}(z)}\right]\left[\overline{\Gamma_{1}(\zeta)+\lambda \Gamma_{2}(\zeta)}\right] d A_{z} d A_{\zeta}\right| \\
& =\mid \int_{G} \int_{G} V(z, \zeta) \overline{\Gamma_{1}(z)} \overline{\Gamma_{1}(\zeta)} d A_{z} d A_{\zeta} \\
& +2 \lambda \int_{G} \int_{G} V(z, \zeta) \overline{\Gamma_{1}(z)} \overline{\Gamma_{2}(\zeta)} d A_{z} d A_{\zeta} \\
& +\lambda^{2} \int_{G} \int_{G} V(z, \zeta) \overline{\Gamma_{2}(z)} \overline{\Gamma_{2}(\zeta)} d A_{z} d A_{\zeta} \mid
\end{aligned}
$$

on the other hand, by (1) and the orthogonality of $\Gamma_{1}$ to $\phi_{n}$ on $G$

$$
\leqq \lambda^{2} \int_{G} \int_{G} K \mathscr{D}(z, \bar{\zeta}) \overline{\Gamma_{2}(z)} \Gamma_{2}(\zeta) d A_{z} d A_{\zeta} \quad \text { for all real } \lambda .
$$

Thus

$$
\int_{G} \int_{G} V(z, \zeta) \overline{\Gamma_{1}(z)} \overline{\Gamma_{1}(\zeta)} d A_{z} d A_{\zeta}=0
$$

and

$$
\int_{G} \int_{G} V(z, \zeta) \overline{\Gamma_{1}(z)} \overline{\Gamma_{2}(\zeta)} d A_{z} d A_{\zeta}=0
$$

Letting 


$$
\Gamma_{2}(\zeta)=\int_{G} V(z, \zeta) \overline{\Gamma_{1}(z)} d A_{z}
$$

by (3)

$$
\begin{aligned}
\int_{G}\left\{\int_{G} V(z, \zeta) \overline{\Gamma_{1}(z)}\right. & {\left.\left[\int_{G} V(z, \zeta) \overline{\Gamma_{1}(z)} d A_{z}\right] d A_{z}\right\} d A_{\zeta}=0 } \\
& \int_{G}\left|\int_{G} V(z, \zeta) \overline{\Gamma_{1}(z)} d A_{z}\right|^{2} d A_{\zeta}=0 .
\end{aligned}
$$

Hence

$$
\int_{G} V(z, \zeta) \overline{\Gamma_{1}(z)} d A_{z}=0 \quad \text { for all } \zeta \text { in } G,
$$

for all functions $\Gamma_{1}(z)$ that are continuous on $G$, have $\int_{G}\left|\Gamma_{1}(z)\right|^{2} d A_{z}$ $<\infty$ and are orthogonal to $\phi_{n}(z)$ on $G$ for $n=1,2, \cdots$.

Let $\delta_{k}\left(z-z_{0}\right)$ be the $k$ th continuous approximation to the delta function at $z_{0}$ such that $\delta_{k}\left(z-z_{0}\right)=0$ for all $z$ in $G$ with $\left|z-z_{0}\right|>1 / k$. Then $\delta_{k}\left(z-z_{0}\right)$ can be expressed by

$$
\Psi_{k}(z)+\sum_{n=1}^{\infty} b_{n}^{(k)} \phi_{n}(z)
$$

for $z$ in $G$, where $\Psi_{k}(z)$ is orthogonal to $\phi_{n}(z)$ for $n=1,2, \cdots$, on $G$, has $\int_{G}\left|\Psi_{k}(z)\right|^{2} d A_{z}<\infty$ and is continuous on $G$. By (4), the definition of $a_{n m}$,

$$
\begin{aligned}
& \int_{G} \int_{G}\left[V(z, \zeta)-\sum_{n, m=1}^{\infty} a_{n m} \phi_{n}(z) \phi_{m}(\zeta)\right] \overline{\left[\Psi_{k}(z)+\sum_{n=1}^{\infty} b_{n}^{(k)} \phi_{n}(z)\right]} \\
& \left.\cdot \overline{\Psi_{k}(\zeta)+\sum_{m=1}^{\infty} b_{m}^{(k)} \phi_{m}(\zeta)}\right] d A_{z} d A_{\zeta}=0 .
\end{aligned}
$$

If $z_{0}$ is in $G$, taking $\lim _{k \rightarrow \infty}$ yields

$$
V\left(z_{0}, z_{0}\right)-\sum_{n, m=1}^{\infty} a_{n m} \phi_{n}\left(z_{0}\right) \phi_{m}\left(z_{0}\right)=0 .
$$

A similar computation using $\delta_{k}\left(z-z_{0}\right)+\delta_{k}\left(z-z_{1}\right)$ for the test function yields

$$
\begin{aligned}
& V\left(z_{0}, z_{0}\right)-\sum_{n, m=1}^{\infty} a_{n m} \phi_{n}\left(z_{0}\right) \phi_{m}\left(z_{0}\right)+V\left(z_{0}, z_{1}\right)-\sum_{n, m=1}^{\infty} a_{n m} \phi_{n}\left(z_{0}\right) \phi_{m}\left(z_{1}\right) \\
& +V\left(z_{1}, z_{0}\right)-\sum_{n, m=1}^{\infty} a_{n m} \phi_{n}\left(z_{1}\right) \phi_{m}\left(z_{0}\right)+V\left(z_{1}, z_{1}\right)-\sum_{n, m=1}^{\infty} a_{n m} \phi_{n}\left(z_{1}\right) \phi_{m}\left(z_{1}\right)=0 .
\end{aligned}
$$


By (5) and the symmetry of $V$ and thus of $a_{n m}$,

$$
V(z, \zeta)=\sum_{n, m=1}^{\infty} a_{n m} \phi_{n}(z) \phi_{m}(\zeta) \quad \text { for all } z \text { and } \zeta \text { in } G .
$$

Hence $V$ is analytic on $\mathcal{G} \times \mathcal{G}$.

It is now shown that the series (6) converge for $z$ and $\zeta$ in $D$.

Let $\theta_{k}(z)+\sum_{n=1}^{\infty} C_{n}^{(k)} \phi_{n}(z)$ be the representation of the $k$ th continuous approximation to the delta function at $z_{0}$ where the representation holds for $z$ in $D$, and $\theta_{k}(z)$ is orthogonal to $\phi_{n}(z)$ on $D$ for $n=1$, $2, \cdots$.

$$
\begin{aligned}
& \left.\mid \int_{D} \int_{D}\left[\sum_{n, m=1}^{L} a_{n m} \phi_{n}(z) \phi_{m}(\zeta)\right] \overline{\left[\theta_{k}(z)+\sum_{n=1}^{\infty} C_{n}^{(k)} \phi_{n}(z)\right.}\right] \\
& \left.\overline{\left[\theta_{k}(\zeta)+\sum_{m=1}^{\infty} C_{m}^{(k)} \phi_{n}(\zeta)\right.}\right] d A_{2} d A_{\zeta} \\
& \left.=\mid \int_{D} \int_{D}\left[\sum_{n, m=1}^{L} a_{n m} \phi_{n}(z) \phi_{m}(\zeta)\right] \overline{\left[\sum_{n=1}^{L} C_{n}^{(k)} \phi_{n}(z)\right.}\right] \\
& \left.\cdot \overline{\sum_{m=1}^{L} C_{m}^{(k)} \phi_{m}(\zeta)}\right] d A_{z} d A_{\zeta} \\
& \left.=\mid \int_{G} \int_{G}\left[\sum_{n, m=1}^{L} Q_{n m} \phi_{n}(z) \phi_{m}(\zeta)\right] \overline{\left[\sum_{n=1}^{L} \frac{C_{n}^{(k)}}{k_{n}} \phi_{n}(z)\right.}\right] \\
& \cdot \overline{\left.\sum_{m=1}^{L} \frac{C_{m}^{(k)}}{k_{m}} \phi_{m}(\zeta)\right]} d A_{z} d A_{\zeta} \\
& \left.=\mid \int_{G} \int_{G} V(z, \zeta) \overline{\left[\sum_{n=1}^{L} \frac{C_{n}^{(k)}}{k_{n}} \phi_{n}(z)\right]} \overline{\left[\sum_{m=1}^{L} \frac{C_{n}^{(k)}}{k_{m}} \phi_{m}(\zeta)\right.}\right] d A_{z} d A_{\zeta} \mid \\
& \left.\leqq \int_{G} \int_{G} K \Phi(z, \bar{\zeta}) \overline{\left[\sum_{n=1}^{L} \frac{C_{n}^{(k)}}{k_{n}} \phi_{n}(z)\right.}\right]\left[\sum_{m=1}^{L} \frac{C_{m}^{(k)}}{k_{m}} \phi_{m}(\zeta)\right] d A_{z} d A_{\zeta} \\
& \left.=\int_{\mathfrak{D}} \int_{\mathscr{D}} K \mathfrak{D}(z, \bar{\zeta}) \overline{\left[\sum_{n=1}^{L} C_{n}^{(k)} \phi_{n}(z)\right.}\right]\left[\sum_{m=1}^{L} C_{m}^{(k)} \phi_{m}(\zeta)\right] d A_{z} d A_{\zeta} \\
& \leqq \int_{\mathscr{D}} \int_{\mathscr{D}} K \mathscr{D}(z, \bar{\zeta})\left[\overline{\theta_{k}(z)+\sum_{n=1}^{\infty} C_{n}^{(k)} \phi_{n}(z)}\right] \\
& \cdot\left[\theta_{k}(\zeta)+\sum_{m=1}^{\infty} C_{m}^{(k)} \phi_{m}(\zeta)\right] d A_{z} d A_{\zeta}
\end{aligned}
$$


taking the $\lim _{k \rightarrow \infty}$

$$
K D\left(z_{0}, \bar{z}_{0}\right) \geqq\left|\sum_{n, m=1}^{L} Q_{n m} \phi_{n}\left(z_{0}\right) \phi_{m}\left(z_{0}\right)\right| \quad \text { for all } L .
$$

A similar computation using a representation of an approximation of the delta function at $z_{0}$ plus the delta function at $z_{1}$ and utilizing (7) yields

$$
K D\left(z_{0}, \bar{z}_{0}\right)+\operatorname{Re} K D\left(z_{0}, \bar{z}_{1}\right)+K D\left(z_{1}, \bar{z}_{1}\right) \geqq\left|\sum_{n, m=1}^{L} a_{n m} \phi_{n}\left(z_{0}\right) \phi_{m}\left(z_{1}\right)\right| .
$$

Hence $\left\{\sum_{n, m=1}^{L} a_{n m} \phi_{n}(z) \phi_{m}(\zeta)\right\}_{L=1}^{\infty}$ is a normal family on $D \times D$. Since it converges to $V(z, \zeta)$ on $G \times G, \sum_{n, m=1}^{\infty} a_{n m} \phi_{n}(z) \phi_{m}(\zeta)$ must converge to an analytic function on $D \times D$ that is a continuation of $V(z, \zeta)$.

Corollary. If $V(z, \zeta)$ is a symmetric, continuous, complex-valued function on $\mathcal{G} \times \mathcal{G}$, and

$$
\left|\sum_{n=1}^{L} \sum_{m=1}^{L} \alpha_{n} \alpha_{m} V\left(z_{n}, z_{m}\right)\right| \leqq \sum_{n=1}^{L} \sum_{m=1}^{L} \alpha_{n} \bar{\alpha}_{m} K \triangleright\left(z_{n}, \overline{z_{m}}\right)
$$

for all complex vectors $\left(\alpha_{1}, \alpha_{2}, \cdots\right)$, and $\left(z_{1}, z_{2}, \cdots\right)$ with all $z_{n}$ in $\mathcal{G}$, then $V(z, \zeta)$ is analytic in $\mathrm{G} \times \mathcal{G}$ and can be continued onto $\mathrm{D} \times \mathrm{D}$.

\section{REFERENCES}

1. Ju. E. Alenicyn, Univalent functions without common values in a multiply connected domain, Trudy Mat. Inst. Steklov. 94 (1968), 4-18= Proc. Steklov Inst. Math. 94 (1968), 1-18. MR 37 \#1579.

2. S. Bergman, The kernel function and conformal mapping, Mathematical Surveys, no. 5, Amer. Math. Soc., Providence, R. I., 1950, pp. 1-18. MR 12, 402.

3. S. Bergman and M. Schiffer, Kernel functions and conformal mapping, Compositio Math. 8 (1952), 205-249. MR 12, 602.

University of California at San Diego, la Jolla, California 92038 